\title{
Localized Quantum Chemistry on Quantum Computers
}

\author{
Matthew Otten,,${ }^{1, *}$ Matthew R. Hermes, ${ }^{2}$ Riddhish Pandharkar, ${ }^{2}$ Yuri Alexeev, ${ }^{3}$ Stephen K. Gray, ${ }^{4, \dagger}$ and Laura Gagliardi ${ }^{5, *}$ \\ ${ }^{1}$ HRL Laboratories, LLC, 3011 Malibu Canyon Road, Malibu, CA 90265 \\ ${ }^{2}$ Department of Chemistry, Pritzker School of Molecular Engineering, James Franck Institute, \\ Chicago Center for Theoretical Chemistry, University of Chicago, Chicago, IL 60637, USA. \\ ${ }^{3}$ Computational Science Division, Argonne National Laboratory, Lemont, IL 60439, USA \\ ${ }^{4}$ Center for Nanoscale Materials, Argonne National Laboratory, Lemont, IL 60439, USA \\ ${ }^{5}$ Department of Chemistry, Pritzker School of Molecular Engineering, \\ James Franck Institute, Chicago Center for Theoretical Chemistry, University of Chicago, \\ Chicago, IL 60637; Argonne National Laboratory, Lemont, IL 60439, USA.
}

(Dated: October 17, 2021)

\begin{abstract}
Quantum chemistry calculations of large, strongly correlated systems are typically limited by the computation cost that scales exponentially with the size of the system. Quantum algorithms, designed specifically for quantum computers, can alleviate this, but the resources required are still too large for today's quantum devices. Here we present a quantum algorithm that combines a localization of multireference wave functions of chemical systems with quantum phase estimation (QPE) and variational unitary coupled cluster singles and doubles (UCCSD) to compute their ground state energy. Our algorithm, termed "local active space unitary coupled cluster" (LAS-UCC), scales linearly with system size for certain geometries, providing a polynomial reduction in the total number of gates compared with QPE, while providing accuracy above that of the variational quantum eigensolver using the UCCSD ansatz and also above that of the classical local active space self-consistent field. The accuracy of LAS-UCC is demonstrated by dissociating $\left(\mathrm{H}_{2}\right)_{2}$ into two $\mathrm{H}_{2}$ molecules and by breaking the two double bonds in trans-butadiene and resources estimates are provided for linear chains of up to $20 \mathrm{H}_{2}$ molecules.
\end{abstract}

\section{INTRODUCTION}

Chemical systems with many close-lying electronic states or, more generally, strongly correlated electrons pose a significant challenge for modern electronic structure theories in computational quantum chemistry ${ }^{1-5}$. When transition metals or heavier elements are involved, degenerate and nearly degenerate electronic states are common, and single-reference electronic structure methods such as Kohn-Sham density functional theory often fail ${ }^{6-8}$. In these situations one has to use multireference methods to generate multiconfigurational wave functions and accurately describe these near degeneracies $^{9-11}$.

Scientists also want to compute properties of large chemical systems or solids with accurate quantum chemistry methods, in spite of steep computational requirements. One way to achieve such computations is to use fragmentation methods. Many variations of fragmentation methods exist ${ }^{12-15}$, but the common feature is that a large molecular system is divided into fragments and quantum-mechanical calculations are performed on the fragments. An especially important case is the application of fragmentation methods to multireference wave functions because of the exponential explosion of the computational cost with respect to the size of the active space of electronic configurations.

In the complete active space self-consistent field (CASSCF) method $^{16}$, all the electronic configurations that can be formed for a given number of active electrons distributed in a given number of active orbitals are included in the wave function. Thus, the wave function scales exponentially with the number of active electrons and orbitals, and the method has only limited application to chemically relevant systems. If one wants to study systems containing, for example, several transition metals ${ }^{17-21}$, the active site of a protein ${ }^{22}$, or extended organic chains in their ground and excited states ${ }^{22,23}$, more affordable multireference methods have to be developed. This is one of the major challenges of modern electronic structure theory.

Reducing the computational cost of CASSCF or other multiconfiguration self-consistent field calculations is pursued both in the development of new well-motivated theoretical approximations and in the application of new developments in computational hardware ${ }^{24,25}$. On the theoretical side, one strategy is to identify subspaces of the CAS that can be treated on different footings ${ }^{26,27}$ or interact with one another only weakly ${ }^{28-32}$. The localized active-space self-consistent field method (LASSCF) is an example of such a strategy ${ }^{33-36}$. LASSCF is designed for applications in which electrons are strongly correlated in different weakly interacting physical regions of a molecule and approximates the strongly correlated part of the wave function as a single antisymmetrized product of subspace wave functions. The computational cost of LASSCF is a linear function of the number of such unentangled subspaces.

Some of the authors have recently shown that LASSCF accurately reproduces the CASSCF spin-state energy gaps of bimetallic compounds and the simultaneous dissociation of two double bonds in bisdiazene at a significantly reduced $\operatorname{cost}^{34,35}$. However, LASSCF fails to recover any electron correlation between fragments, for example in the cis-trans isomerization of stilbene and similar systems ${ }^{36}$. Moreover, because the wave function of each fragment is a general manybody wave function, it is not straightforward ${ }^{10,37}$ to apply traditional perturbative or truncated coupled-cluster (CC) corrections based on second quantization ${ }^{38,39}$ on top of a LASSCF reference to recover the missing correlation, at least on classical computational hardware. 
Recently, the development of quantum computers has led to an increased interest in novel quantum algorithms, especially for computational quantum chemistry, which is widely seen as a potential "killer app" of quantum computers ${ }^{40-42}$. Some quantum algorithms for quantum chemistry, such as the quantum phase estimation (QPE) ${ }^{43}$ and unitary coupled cluster (UCC $)^{44,45}$, offer exponential speedups when large faulttolerant quantum computers are available ${ }^{46,47}$. For the noisy, intermediate-scale quantum (NISQ) ${ }^{48}$ devices that we have today, these algorithms are not tenable, since they require coherence times far beyond what is available. Variational algorithms, such as the variational quantum eigensolver $(\mathrm{VQE})^{45}$, have been used to perform calculations of the ground state energy of small molecules, with limited accuracy, on NISQ devices $^{49-51}$. Quantum algorithms that have less stringent requirements compared with full QPE, and at the same time accuracy beyond that demonstrated by variational algorithms such as VQE, will be required to productively use the progressively larger and higher-quality quantum devices as they become available in the next few years.

In this paper we describe a framework for such quantum algorithms, inspired by classical LASSCF. The wave function within a fragment is solved by using one method (e.g., QPE), and correlation between fragments is encoded variationally by using an ansatz that entangles the fragments. This approach goes beyond what can be achieved with classical fragment methods, such as LASSCF, by providing additional correlation between fragments, while significantly reducing the total computational time (estimated via the number of gates) compared with full QPE.

\section{THEORY}

\section{A. Multireference Methods with Exponential Scaling}

We seek to find the ground state of the second-quantized molecular Hamiltonian for a given number of $M$ electrons,

$$
\hat{H}=h_{q}^{p} \hat{a}_{p}^{\dagger} \hat{a}_{q}+\frac{1}{4} h_{q s}^{p r} \hat{a}_{p}^{\dagger} \hat{a}_{r}^{\dagger} \hat{a}_{s} \hat{a}_{q},
$$

where $\hat{a}_{p}^{\dagger}\left(\hat{a}_{p}\right)$ creates (annihilates) an electron in spin orbital $p ; h_{q}^{p}$ and $h_{q s}^{p r}$ are the one- and antisymmetrized two-electron Hamiltonian matrix elements, respectively; and repeated internal indices are summed. Generally, for $N$ spin orbitals, $\hat{H}$ has a sparse-matrix representation in a space of size $O\left(\begin{array}{l}N \\ M\end{array}\right)$ and has $O\left(N^{4}\right)$ elements. Full-configuration interaction (FCI) determines the exact energy within a given one-electron basis set (the FCI energy) at exponential cost. Methods such as CASSCF (and its restricted ${ }^{52,53}$ and generalized ${ }^{54,55}$ active space approximations) or selected configuration interaction $^{56,57}$, can go beyond FCI in system size, maintaining comparable accuracy, but still scale exponentially. The density matrix renormalization group ${ }^{58-61}$ and coupled cluster methods $^{39}$ can scale polynomially but introduce (sometimes uncontrollable) approximation errors. Here we briefly describe the LASSCF algorithm ${ }^{33,35}$, which will serve as the basis for our fragment-based quantum algorithms.

\section{B. LASSCF}

In LASSCF, the wave function of a molecule is approximated as

$$
|\mathrm{LAS}\rangle=\bigwedge_{K}\left|\Psi_{K}\right\rangle \wedge|\Phi\rangle,
$$

where $\left|\Psi_{K}\right\rangle$ is a general many-body wave function describing $M_{K}$ electrons occupying $N_{K}$ active orbitals of the $K$ th "fragment" or "active subspace," $|\Phi\rangle$ is a single determinant spanning the complement of the complete active space, and the wedge operator (“ $\wedge$ ”) implies an antisymmetrized product.

In the variational ${ }^{35}$ implementation of LASSCF, this wave function is obtained by minimizing the LAS energy,

$$
E_{\mathrm{LAS}}=\langle\mathrm{LAS}|\hat{H}| \mathrm{LAS}\rangle,
$$

with respect to all orbital rotations and configuration interaction (CI) vectors defining $|\mathrm{LAS}\rangle$. This is accomplished by introducing a unitary operator (see the Supporting Information of Ref. 35) that is parameterized in terms of all nonredundant transformations of the orbitals and CI vectors,

$$
|\mathrm{LAS}\rangle \rightarrow \hat{U}_{\mathrm{orb}} \prod_{K} \hat{U}_{\mathrm{Cl}, K}|\mathrm{LAS}\rangle,
$$

where

$$
\begin{aligned}
\hat{U}_{\text {orb }} & =x_{l}^{k}\left(\hat{a}_{k}^{\dagger} \hat{a}_{l}-\hat{a}_{l}^{\dagger} \hat{a}_{k}\right), \\
\hat{U}_{\mathrm{Cl}, K} & =x_{\vec{k}}\left(|\vec{k}\rangle\left\langle\Psi_{K}|-| \Psi_{K}\right\rangle\langle\vec{k}|\right),
\end{aligned}
$$

where $k, l$ index individual spin orbitals in two different subspaces (including the inactive and virtual subspaces outside of the CAS) and where $|\vec{k}\rangle$ is a determinant or configuration state function. First and second derivatives of Eq. (3) with respect to the generator amplitudes $\left(x_{l}^{k}\right.$ and $\left.x_{\vec{k}}\right)$ are obtained by using the Baker-Campbell-Hausdorff $(\mathrm{BCH})$ expansion, and the energy is minimized by repeated applications of the preconditioned conjugate gradient (PCG) method ${ }^{62,63}$.

The orbital unitary operator, $\hat{U}_{\text {orb }}$, corresponds to the UCC correlator truncated after the first ("singles") term:

$$
\begin{aligned}
& \hat{U}_{\mathrm{UCC}} \equiv \exp \left\{\hat{T}_{\mathrm{UCC}}\right\}, \\
& \hat{T}_{\mathrm{UCC}} \equiv x_{l}^{k}\left(\hat{a}_{k}^{\dagger} \hat{a}_{l}-\text { h.c. }\right)+\frac{1}{4} x_{l n}^{k m}\left(\hat{a}_{k}^{\dagger} \hat{a}_{m}^{\dagger} \hat{a}_{n} \hat{a}_{l}-\text { h.c. }\right)+\ldots
\end{aligned}
$$

In principle, the augmentation of the orbital rotation unitary operator, Eq. (5), by the second term of the more general cluster operator, Eq. (8), could encode electron correlation and entanglement between active subspaces into the LAS wave function. This would require the reference wave function, $|\mathrm{LAS}\rangle$, to be updated by explicit exponentiation of the general cluster operator, Eq. (8), after each execution of the PCG algorithm. On classical computer hardware, however, this is not an efficient way to extend LASSCF.

\section{LAS Methods on Quantum Computers}

Here we describe an algorithm for molecular calculations that goes beyond the limited accuracy of standard $\mathrm{VQE}^{50,51}$, 
while having dramatically reduced computational complexity compared with QPE [see Methods section]. The algorithm exploits the structure of the molecule by separating it into coupled fragments, as is done in the classical algorithm, LASSCF. The quantum algorithm, however, goes beyond classical LASSCF by providing some degree of entanglement between the fragments.

The algorithm begins by segmenting the orbital active space of a given molecule into distinct fragments defined by nonoverlapping orbital subspaces, as in classical LASSCF. For instance, orthogonalized atomic orbitals generated by using the meta-Löwdin method ${ }^{64}$ can be sorted into localized fragments and then projected onto a guess for the CAS of a given molecule to produce localized active orbitals. We construct an effective Hamiltonian that omits non-mean-field interfragment interactions, resulting in a sum of local fragment Hamiltonians,

$$
\hat{H}_{\mathrm{eff}}=\sum_{K}^{n_{f}}\left(\tilde{h}_{k_{2}}^{k_{1}} \hat{a}_{k_{1}}^{\dagger} \hat{a}_{k_{2}}+\frac{1}{4} h_{k_{2} k_{4}}^{k_{1} k_{3}} \hat{a}_{k_{1}}^{\dagger} \hat{a}_{k_{3}}^{\dagger} \hat{a}_{k_{4}} \hat{a}_{k_{2}}\right)
$$

where $k_{1}, k_{2}, \ldots$ index distinct active orbitals of the $K$ th fragment and where

$$
\tilde{h}_{k_{2}}^{k_{1}}=h_{k_{2}}^{k_{1}}+h_{k_{2} i}^{k_{1} i}+\sum_{L \neq K} h_{k_{2} l_{2}}^{k_{1} l_{1}} \gamma_{l_{2}}^{l_{1}},
$$

where $i$ and $l_{n}$ index respectively inactive orbitals [i.e., those defining $|\Phi\rangle$ in Eq. (2)] and active orbitals of the $L$ th fragment and where $\gamma_{l_{2}}^{l_{1}}$ is a density matrix element for spin orbitals $l_{1}$ and $l_{2}$.

Given a set of localized active orbitals that minimize the LASSCF energy, if the density matrices in Eq. (10) are obtained from a classical LASSCF calculation on the same system, then the QPE algorithm applied to $\hat{H}_{\text {eff }}$ generates the active-space part of the LASSCF wave function, $|\mathrm{QLAS}\rangle=$ $\bigwedge_{K}\left|\Psi_{K}\right\rangle$, on the quantum computer. The same result is achieved if density matrices are obtained self-consistently from the QPE evaluation. If the density matrices are obtained in some other way, for instance from $|\mathrm{HF}\rangle$, then an approximation to the LASSCF wave function is obtained.

A sequence of UCC with singles and doubles (UCCSD) circuits, with variable parameters, is then applied across $m$ fragments each (which we term $m$-local), leading to the LAS-UCC wave function,

$$
|\mathrm{QLAS}\rangle \rightarrow \prod_{L} \hat{U}_{\mathrm{UCCSD}, L}|\mathrm{QLAS}\rangle,
$$

where $\hat{U}_{\mathrm{UCCSD}, L}$ is the UCCSD ansatz including only creation/annihilation operators within the $m$ fragments that it spans. The parameters of the UCCSD circuit are varied to minimize the total energy of the full system, as in VQE [see Methods section]. A schematic representation of the described circuit is shown in Fig. 1. This provides electron correlation between the fragments, in a way that scales exponentially on classical computers, but only polynomially on quantum computers. Moreover, this procedure provides a better estimate of the ground state energy than the product wave

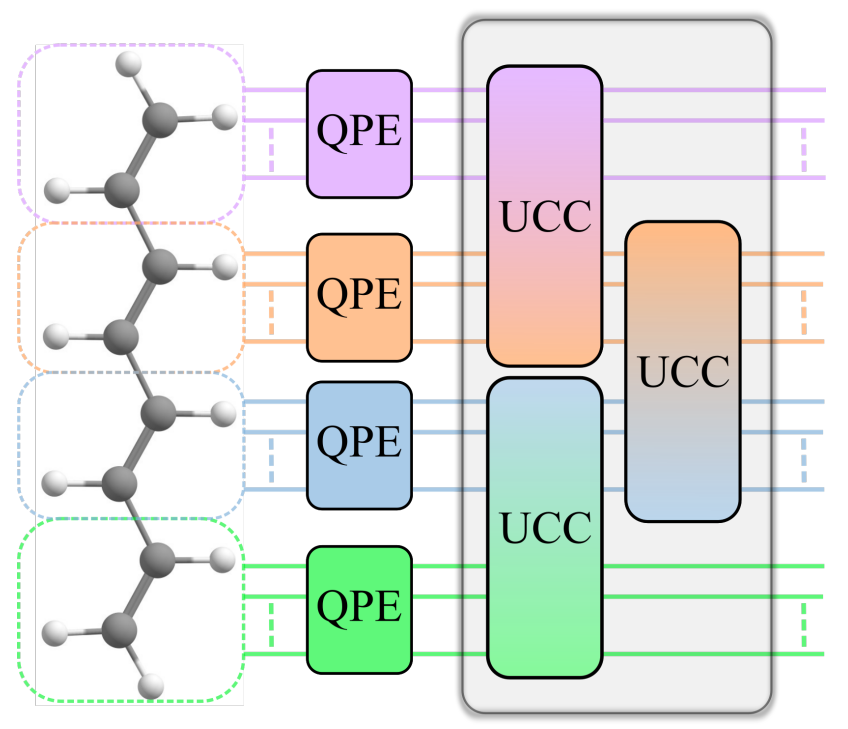

FIG. 1. Diagram of example circuit using LAS-UCC. The system of interest is first separated into distinct fragments. QPE is used on each fragment to solve for the approximate unentangled ground state. Correlation between fragments is then added in, variationally, through a unitary coupled cluster ansatz.

function or the UCCSD would provide alone. Note that, unlike LASSCF, this method is not strictly variational (despite the use of VQE) because the initial product-state wave function, $\bigwedge_{K}\left|\Psi_{K}\right\rangle$, is not variationally reoptimized in the presence of the UCCSD correlators. The QPE circuits could also be replaced with a local variational ansatz, leading to a fully variational algorithm, which we term LAS-VQE and describe in the Supplementary Information.

To understand the large improvement in computational complexity of our approach, we focus on a system of $n_{f}$ fragments, with the number of orbitals per fragment, $N_{K}$, constant as the number of fragments grows. The total system size is defined by $N=N_{K} n_{f}$ orbitals. We also assume that each fragment interacts with only the $m$ geometrically nearest fragments and that $m$ does not grow with $n_{f}$. These are reasonable assumptions for many interesting molecules and mirror the assumptions made in classical LASSCF. Under these assumptions, the QPE solver for the unentangled fragments does not grow with $N$, since $N_{K}$ is assumed to be fixed while $n_{f}$ grows. The number of small QPE sections grows linearly with the number of fragments, of course. Typically, the JordanWigner transformation would introduce an $O(N)$ term to enforce the anticommutation relations among the orbital creation and annhilation operators. However, in the case of linear chains, as we study here, ordering the orbitals such that all up and down occupied and virtual orbitals in a given fragment are close, the high-weight $Z$ part of the Jordan-Wigner transformation effectively cancels out, causing no scaling with total number of orbitals. See Supplementary Information for more details. Together, this leads to an overall $O\left(n_{f} N_{K}^{4}\right) \approx O(N)$ (linear) number of gates to solve for the $n_{f}$ unentangled product wave functions. The UCCSD correlator, which is then applied, has $O\left(m^{4} N_{K}^{4}\right)$ terms in the cluster operator for each cor- 


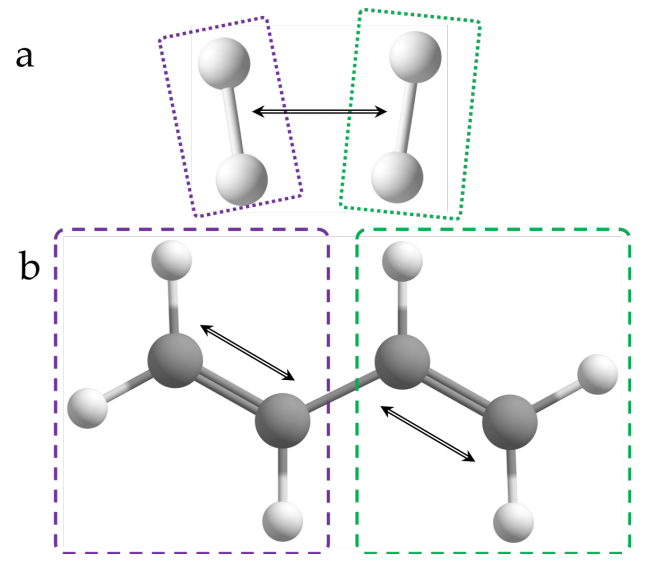

FIG. 2. Two model systems used for testing. (a) The asymmetric hydrogen dimer, $\left(\mathrm{H}_{2}\right)_{2}$. Each $\mathrm{H}_{2}$ molecule is a fragment described by a 2-electron, 2-spatial orbital or $(2,2)$ active subspace in the dimer's LAS wave function. The potential energy surface is scanned along the distance between the two $\mathrm{H}_{2}$ bond midpoints, indicated by the black double line. (b) The trans-butadiene molecule at its CASSCF $(8,8) / 6-31 \mathrm{G}$ ground-state equilibrium geometry. Dashed boxes depict the two notional fragments containing the two $(4,4)$ active subspaces in the LAS wave function. Black double lines indicate the internal coordinate along which the potential energy surface is scanned; the two terminal methylene units are simultaneously removed from the central acetylene unit.

relator, because the UCCSD circuit spans only $m$ fragments. Neither $m$ nor $N_{K}$ grows with the total size (number of spin orbitals) of the system, $N$. The number of $m$-local correlators grows as $O\left(n_{f}\right)$. Again, by careful ordering of the orbitals, the Jordan-Wigner transformation does not introduce any scaling overhead. The complexity of the $m$-local UCCSD correlator is then $O\left(n_{f} m^{4} N_{K}^{4}\right) \approx O(N)$ (linear). This creates an overall linear scaling in the number of gates for linear chain geometries, with respect to only the total size of the system, $N$, and is polynomially $\left(O\left(N^{4}\right)\right)$ better than performing QPE alone, while providing accuracy above VQE using the UCCSD ansatz and classical LASSCF. Many of the gates can be done in parallel, such as the local QPE circuits and the different $m$-local UCCSD correlators, leading to an expected overall sub-linear depth. If the fragments are coupled in a geometry more complicated than a linear chain, the UCCSD correlator will potentially incur the $O(N)$ Jordan-Wigner overhead, leading to an overall $O\left(N^{2}\right)$ scaling for arbitrary geometries with an expected $O(N)$ depth.

\section{Illustrative Molecular Systems}

In the calculations discussed below, we consider two systems, depicted in Fig. 2. The first, shown in Fig. 2(a), is a simplistic model of weakly interacting fragments, consisting of two $\mathrm{H}_{2}$ molecules at various distances between their two midpoints using a minimal STO-3G atomic orbital (AO) basis set, and the two active subspaces in the LAS wave function correspond to the active spaces of the two $\mathrm{H}_{2}$ molecules. We use this small basis set because of the size limitations of today's quantum computers and simulations. The bond lengths and internal angles of this system are set arbitrarily to remove point group symmetry so that differences between various methods are not obscured by the simplicity of a symmetrized electronic wave function. The interaction between the two fragments in this model system are weak, and the LAS wave function is therefore expected to provide an excellent model of the FCI wave function except when the distance between the two molecules is very small. We additionally extend this system up to $20 \mathrm{H}_{2}$ in a linear chain, where we estimate only the total number of quantum resources necessary.

The second system, depicted in Fig. 2(b), is the transbutadiene molecule. The potential energy surface of this molecule is scanned along the internal coordinate corresponding to the simultaneous stretching of both the $\mathrm{C}=\mathrm{C}$ double bonds, leading to the removal of two methylene units from a central $\mathrm{C}_{2} \mathrm{H}_{2}$ (distorted acetylene-like) unit. In the LAS wave function, the molecule is divided into two fragments split across the central $\mathrm{C}-\mathrm{C}$ bond, and each fragment is described by a $(4,4)$ active subspace. Several molecular orbitals are therefore left inactive, described by an unfragmented single determinant. We employed the 6-31G AO basis set in this case.

The trans-butadiene system is a chemical model of the case of two strongly interacting units in a system, where the value of the stretching internal coordinate is a proxy for the strength of electron correlation. Near the equilibrium geometry, dividing the active space into two fragments is chemically reasonable: each fragment encloses one $\pi$-bond, and inasmuch as electron correlation affects the system at all, it is a reasonable approximation to consider it only locally. However, as the $\mathrm{C}=\mathrm{C}$ double bonds are elongated, electrons from the two broken $\pi$ bonds recouple across the central $\mathrm{C}_{2} \mathrm{H}_{2}$ unit, which spans the fissure between the two LAS fragments. The LAS wave function cannot model a $\pi$ bond in this position, and the LASSCF method breaks down.

\section{RESULTS AND DISCUSSION}

\section{A. LAS-UCC}

We demonstrate the efficacy of our framework by simulating the two benchmark molecules, $\left(\mathrm{H}_{2}\right)_{2}$ and trans-butadiene, described above. We compare three methods: LASSCF, CAS configuration interaction in the basis of LASSCF orbitals (CASCI), and our new algorithm, LAS-UCC. LASSCF represents the best unentangled set of wave functions and is equivalent to the solution after the QPE circuits but before the use of the UCCSD ansatz. Note that CASCI is slightly different from CASSCF since the orbitals are not variationally reoptimized. CASCI solves for the FCI wave function within the active space; in this case, it is equivalent to using QPE across the whole molecule and represents the reference result in these studies.

Figure 3 shows the results of applying the methods to the hydrogen dimer as the two $\mathrm{H}_{2}$ molecules are pulled apart. We 


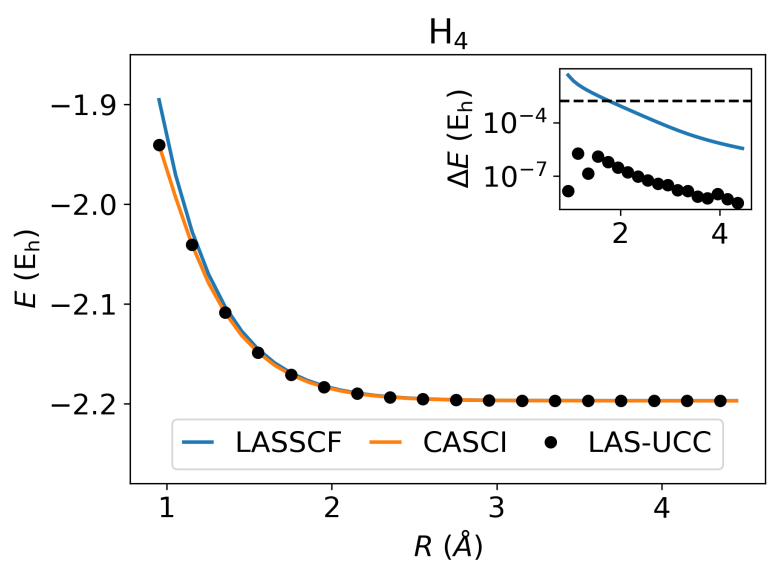

FIG. 3. Energies for $\left(\mathrm{H}_{2}\right)_{2}$ calculated by CASCI, LASSCF, and LAS-UCC. The inset shows the error, with respect to CASCI, of LASSCF and LAS-UCC. The black dashed line represents chemical accuracy. LAS-UCC is able to obtain chemical accuracy, with respect to CASCI, at all distances. LASSCF cannot obtain chemical accuracy at sufficiently short distances.

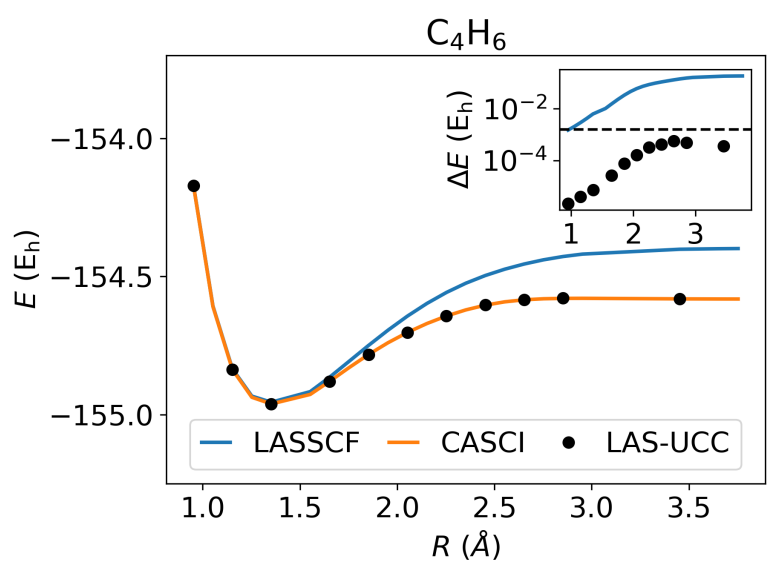

FIG. 4. Energies for $\mathrm{C}_{4} \mathrm{H}_{6}$ calculated by CASCI, LASSCF, and LASUCC. The inset shows the error, with respect to CASCI, of LASSCF and LAS-UCC. The black dashed line represents chemical accuracy. LAS-UCC obtains chemical accuracy across the potential energy surface, whereas LASSCF, which cannot accurately represent the correlation between the fragments, fails to obtain chemical accuracy for most points.

see that LASSCF, CASCI, and LAS-UCC agree except for very small distances where LASSCF no longer provides accurate energies.

Figure 4 shows the results for trans-butadiene, a model of strongly correlated fragments. Here, as the terminal methylene units are removed, the interfragment correlation grows as a double bond is formed between the fragments. The UCCSD ansatz can accurately represent this level of entanglement, allowing LAS-UCC to achieve nearly CASCI accuracy, whereas LASSCF fails to account for this entanglement.

\section{B. Resource Estimates}

To demonstrate the scaling advantage of our method, we perform resource estimation for the number of logical quantum gates necessary for several different quantum algorithms: the QPE algorithm over the full unfragmented molecule; the UCCSD ansatz over the full unfragmented molecule; and the two steps of our proposed LAS-UCC method, the fragmented QPE and the 2-local UCCSD (which corresponds to the circuit depicted in Fig. 1). We estimate the number of resources needed for the QPE algorithm if only a single Trotter time step were needed; $O(1000)$ time steps will be needed for typical systems to get to chemical accuracy ${ }^{65,66}$. Note that these estimates represent only the number of two-qubit CNOT gates, which we use as a primary gauge of the number of total resources. Single-qubit gates are also necessary; the estimates for these resources can be found in the Supplementary Information and scale similarly to the number of CNOT gates.

We use a model system of an increasing number of $\mathrm{H}_{2}$ molecules and look at how the number of CNOT gates increases as the number of molecules increases, as shown in Fig. 5. As the number of $\mathrm{H}_{2}$ molecules increases, the number of gates needed for all methods also increases. As predicted in the complexity analysis of QPE [see Methods section], the total number of gates for a single Trotter step in the QPE algorithm grows as $O\left(N^{5}\right)$. Similarly, the number of gates needed for a global UCCSD ansatz also grows as $O\left(N^{5}\right)$, as expected ${ }^{50}$. This result is compared with the much smaller number of gates necessary to implement the two steps of our LAS-UCC algorithm. As expected, both the QPE and UCCSD parts of LAS-UCC provide dramatic scaling advantages, with the 2-local UCCSD ansatz and the QPE of the reduced Hamiltonian both scaling as only $O(N)$. We note that, in addition to evaluating the quantum circuits here, an additional optimization loop is needed when using the UCCSD ansatz, whether it is global or 2-local. Using a 2-local UCCSD ansatz also greatly reduces the number of parameters that need to be optimized compared with a global UCCSD ansatz.

\section{Discussion}

Here we compare LAS-UCC with the two quantum algorithms that it is composed of: QPE and variational UCCSD. Compared with global QPE, LAS-UCC reduces the total quantum resource cost by approximating the system with noninteracting fragments and adding in some interaction between fragments (those described by a UCCSD ansatz spanning the fragments). This in general reduces the accuracy; but as shown in the preceding sections, LAS-UCC provides accuracy comparable to CASCI (and therefore global QPE) for the systems considered here. The trans-butadiene molecule is a model for larger, more complicated systems of strongly interacting units. Many single molecular magnets have such pockets of strong correlation localized on the metal centers, which moderately interact with each other ${ }^{67,68}$. With LASUCC we not only can obtain the wave function efficiently but also can selectively couple the fragments with the UCC corre- 


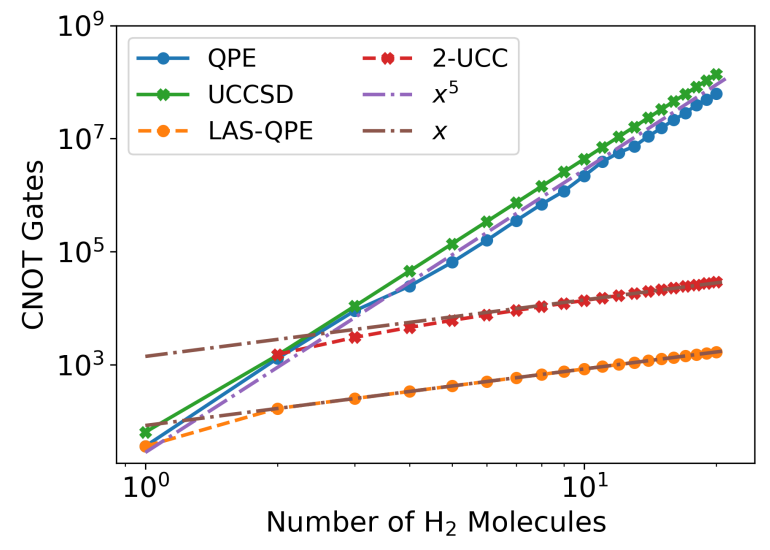

FIG. 5. Estimated two-qubit gate counts using various algorithms. The QPE estimates assume only a single Trotter step. Polynomials of various orders have been plotted to demonstrate the scaling. Our algorithm, LAS-UCC, requires both the LAS-QPE and 2-UCC circuits and thus has an overall $O(N)$ scaling, compared with the $O\left(N^{5}\right)$ scaling of UCC and QPE.

lator, offering further insight into the nature of these interactions. Affordable and accurate modeling of phenomena such as singlet fission ${ }^{69,70}$ in molecular crystals of conjugated organic compounds can be performed with LAS-UCC, as faulttolerant quantum computers become available. This approach will also be used to study chemical processes involving interfragment bond formation and breaking while still treating all points on a potential energy surface at comparable footing.

Compared with standard UCCSD, LAS-UCC can be seen as augmenting UCCSD with a multireference initial state. Instead of using single-determinant Hartree-Fock, as is standard in VQE demonstrations of UCCSD ${ }^{51,71-74}$, LAS-UCC uses the unentangled product state of the ground state wave functions of each fragment (which is also the LASSCF wavefunction). This provides additional accuracy, above standard single-reference UCCSD, at a negligible increase in cost. When using a global UCCSD ansatz, the increase in the number of gates is negligible, even when taking into account the $O(1000)$ time steps that would be needed to implement the QPE step. Using the $m$-local ansatz provides further reduction.

Moreover, recent advances in VQE algorithms have developed various ways to reduce the cost associated with the UCC correlator $^{40,42,75-78}$. As presented in the Theory section, LASUCC can also be seen as a post-LASSCF method that recouples select fragments at a level of theory beyond the mean field. The addition of the doubles or higher terms in the cluster operator provides a way to systematically improve the accuracy beyond the LASSCF reference. Such an implementation of UCC on classical computers cannot be done efficiently beyond first order.

Not every system will be accurately described by LASUCC, of course, but one can systematically increase the accuracy in several ways, while increasing the total resource cost.
Increasing the size of each fragment (which in turn decreases the number of fragments) gradually increases the accuracy, until the limit of a single fragment, where the UCCSD ansatz becomes redundant and the algorithm becomes simply global QPE. On the UCC side, the order of the ansatz can be increased. Triples, quadruples, and so on can be included at increasing cost. If using an $m$-local ansatz, the scaling is unaffected, but the total number of gates increases. The locality of the ansatz, $m$, can also be increased, providing explicit correlation between more geometrically distant fragments.

\section{CONCLUSIONS}

We introduced for the first time LAS-UCC, a quantum algorithm that combines a fragmentation of the wave function of a chemical system with QPE and variational UCCSD to compute the ground state energy of such a system. LASUCC can describe compounds containing strongly interacting fragments, and it provides a polynomial scaling advantage in the number of quantum gates compared with other quantum algorithms such as QPE and UCCSD. Since the fragments' reduced Hamiltonians have fewer terms and by ensuring the locality of the Jordan-Wigner transform, the overall gate count will be $O(N)$ with respect to the total size of the system $N$ for for linear geometries and $O\left(N^{2}\right)$ more generally, compared with $O\left(N^{5}\right)$ requirements for QPE. We also demonstrated the accuracy of LAS-UCC on $\left(\mathrm{H}_{2}\right)_{2}$ and transbutadiene molecules and performed resource estimations of larger systems to demonstrate the scaling advantage.

As larger fault-tolerant quantum computers are developed, we expect that our algorithm will be able to provide accurate calculations of large and useful chemical systems, such as molecular magnets and qubits, photovoltaic materials, and large biomolecules that are out of reach of classical computing algorithms but for which QPE would be too expensive.

\section{METHODS}

\section{A. Quantum Algorithms}

Here we describe two quantum algorithms that serve as the primary components for our fragment-based quantum algorithm.

\section{Quantum Phase Estimation}

The quantum phase estimation algorithm solves for the eigenvalue, $\lambda_{k}$, for an eigenvector $\left|v_{k}\right\rangle$ of some unitary matrix, $U$. In addition to its use in quantum chemistry, it forms the basis for many important quantum algorithms, such as Shor's prime number factoring algorithm ${ }^{79}$ and the HassidimHarrow-Lloyd algorithm for inverting matrices ${ }^{80}$. For quantum chemistry problems, the unitary matrix $U$ is generated by 
the Hamiltonian, $H$ (eq. (1)), over time steps $\tau$ :

$$
U\left|v_{k}\right\rangle=e^{-i \hat{H} \tau}\left|v_{k}\right\rangle=e^{i 2 \pi \phi}\left|v_{k}\right\rangle
$$

and the desired energy is mapped to the phase acquired, $E=$ $-2 \pi \phi / \tau$, where units have been chosen such that $\hbar=1$. By combining real-time evolution of the Hamiltonian, $\hat{H}$, with application of the quantum Fourier transform $(\mathrm{QFT})^{81,82}$, the value of the energy can be obtained in polynomial time using a quantum computer.

The computational complexity of the QPE is directly related to the complexity of implementing the unitary propagator $U=e^{-i \hat{H \tau}}$. Many strategies for implementing $U$ exist, including Trotterization ${ }^{83,84}$, Taylorization ${ }^{85}$, and qubitization ${ }^{86}$. The Hamiltonian, Eq. (1), has $O\left(N^{4}\right)$ terms, where $N$ is the number of spin orbitals. Each term in the Hamiltonian can be transformed into a Pauli string (that is, a product of Pauli operators $X, Y, Z$, or $I$ ) via one of the many fermion-tospin transformations, such as the Jordan-Wigner ${ }^{87}$, parity ${ }^{88}$, and Bravyi-Kitaev ${ }^{89}$ transformations. In this work we focus on QPE using Trotterization with the Jordan-Wigner transformation since they serve as standard reference points for the other variations. The complexity of QPE for the Hamiltonian, Eq. (1), using Trotterization with the Jordan-Wigner transformation is $O\left(N^{5}\right): N^{4}$ arising from the number of terms in the Hamiltonian and an additional $N$ from the Jordan-Wigner transform. Although QPE can obtain estimates of the ground state energy with only a polynomial number of quantum gates, the overheads are still too large for near-term quantum computers. The success of the QPE algorithm directly depends on the overlap of the initial state (which is often taken to be the Hartree-Fock state) and the true ground state. Realistic estimates, taking into account overheads such as quantum error correction, put the needed number of qubits to perform QPE on interesting molecules in the millions ${ }^{90-92}$.

QPE is analogous to a Fourier analysis of a correlation function; and, for a given energy accuracy, $\epsilon$, it requires propagation efforts (maximum times) on the order of $O(1 / \epsilon)^{65,66}$. Since the circuit depth for evaluating the propagator for individual fragments will naturally be lower than for the full system, the QPEs involved in our LAS approach will be significantly cheaper than full QPE.

\section{Variational Quantum Eigensolver}

The variational quantum eigensolver is a hybrid quantumclassical algorithm that relies on the variational principle to find an estimate of the ground state energy of a given molecule. A circuit with variable parameters, $\theta$, serves as an ansatz, whose energy is evaluated on a quantum computer and whose parameters are iteratively optimized by a classical computer. For a circuit ansatz $|\psi(\theta)\rangle$, VQE estimates the energy as

$$
E=\min _{\theta}\langle\psi(\theta)|\hat{H}| \psi(\theta)\rangle .
$$

The Hamiltonian, $\hat{H}$, is transformed into a sum of Pauli strings via a fermion-to-spin transformation, and the expectation value of each term is measured from the quantum computer separately and summed on the classical computer. VQE has much less stringent quantum resource requirements than QPE has, since it offloads much of the work (such as optimization) to the classical computer. Hence, VQE has been used in proof-of-principle calculations for small molecules ${ }^{71,73,74}$.

The accuracy of VQE is determined by the quality of the ansatz, $|\psi(\theta)\rangle$. The UCCSD ansatz is an interesting choice as wave function for VQE since there is no known way to efficiently implement UCCSD on classical computers ${ }^{93-95}$, but it can be implemented with $O\left(N^{5}\right)$ gates on quantum computers $^{50,96,97}$. The UCCSD ansatz is

$$
\left|\psi_{\mathrm{UCCSD}}\right\rangle=\hat{U}_{\mathrm{UCCSD}}|\mathrm{HF}\rangle=\exp \left\{\hat{T}_{\mathrm{UCCSD}}\right\}|\mathrm{HF}\rangle,
$$

where $\hat{T}_{\mathrm{UCCSD}}$ is defined by truncating the more general cluster operator of Eq. (8) at the second term. While the UCCSD ansatz can be implemented on NISQ devices for small molecules ${ }^{51,72}$, it is limited in its accuracy because of only including up to doubles excitations.

\section{B. Computational Methods}

To calculate the accuracy of the proposed method for small molecules, we use the following strategy. We first use a classical LASSCF solver, as implemented in the $m r h$ package $^{98}$, to find the best product wave function. This effectively provides an equivalent solution to that of the QPE step of our proposed algorithm. We then represent this product wave function as a CI vector in the complete active Fock space and apply a UCCSD correlator, as well as its derivatives with respect to all amplitudes, to this reference CI vector. We employ the factorization reported by Chen et al. ${ }^{99}$ to avoid the $\mathrm{BCH}$ expansion and its inevitable approximate truncation. The resulting $|\mathrm{QLAS}\rangle \mathrm{CI}$ vector and its derivatives ( $|\delta \mathrm{QLAS}\rangle)$ with respect to the unitary coupled cluster amplitudes are used to compute the energy, $\langle\mathrm{QLAS}|\hat{H}| \mathrm{QLAS}\rangle$, and its derivatives, $\langle\delta \mathrm{QLAS}|\hat{H}| \mathrm{QLAS}\rangle$. We then minimize the former using the latter and the Broyden-Fletcher--Goldfarb--Shanno algorithm. We find that this approach is more efficient than directly simulating the quantum circuits. We note that this method scales exponentially on classical computers.

To provide gate count estimates, we use the $\mathrm{Q} \#$ package ${ }^{100}$, generally following the framework of Ref. 101. The full and reduced Hamiltonians are produced by using the $m r h$ package $^{98}$, and both Hamiltonians are then passed to the Q\# package to estimate the number of CNOT gates using the QPE algorithm with a single Trotter time step for each. Additionally, we estimate the number of CNOT gates necessary to calculate various UCCSD ansatzes, including a global UCCSD ansatz over the whole unfragmented molecule and multiple 2local ansatzes that span only two fragments. We count only the number of logical quantum gates needed. Real quantum computers will require additional overheads, owing to limited connectivity and the need to use expensive quantum error correction protocols to deal with inevitable errors ${ }^{91,92}$. Furthermore, we provide gate counts only; no attempt was made to count gate depth, which is typically smaller, because many gates can be implemented in parallel. 


\section{CONTRIBUTIONS}

L.G., S.G. M.O. and M.R.H. designed the project. M.O. wrote the quantum algorithm and tested it. M.R.H. wrote the LASSCF classical code. R.P. tested the codes and performed some of the calculations Y.A. helped with the theory and suggested testing calculations. M.O. and M.R.H. wrote the initial draft of the manuscript. All authors contributed to the scientific discussions and manuscript revisions.

\section{ACKNOWLEDGMENTS}

This research is based on work supported by Laboratory Directed Research and Development (LDRD) funding from Argonne National Laboratory, provided by the Director, Office of Science, of the U.S. DOE under Contract No. DE-
AC02-06CH11357. This work was performed, in part, at the Center for Nanoscale Materials, a U.S. Department of Energy Office of Science User Facility, and supported by the U.S. Department of Energy, Office of Science, under Contract No. DE-AC02-06CH11357. MRH and LG are partially supported by the U.S. Department of Energy (DOE), Office of Basic Energy Sciences, Division of Chemical Sciences, Geosciences, and Biosciences under grant no. USDOE/DESC002183. This material is based upon work supported by the U.S. Department of Energy, Office of Science, National Quantum Information Science Research Centers. We gratefully acknowledge the computing resources provided on Bebop, a high-performance computing cluster operated by the Laboratory Computing Resource Center at Argonne National Laboratory and University of Chicago Research Computing Center. *mjotten@hrl.com

$\dagger$ gray@anl.gov

¥ lgagliardi@uchicago.edu

1 P.-O. Löwdin, Adv. Chem. Phys. , 207 (1958).

2 C. D. Sherrill, A. Dutta, M. L. Abrams, and J. S. Sears (ACS Publications, 2007).

3 A. I. Krylov, L. V. Slipchenko, and S. V. Levchenko, in ACS Symposium Series, Vol. 958 (Citeseer, 2007) pp. 89-102.

${ }^{4}$ T. Stein, T. M. Henderson, and G. E. Scuseria, J. Chem. Phys. 140, 214113 (2014).

5 C. A. Gaggioli, S. J. Stoneburner, C. J. Cramer, and L. Gagliardi, ACS Catal. 9, 8481 (2019).

${ }^{6}$ F. Neese, Coord. Chem. Rev 253, 526 (2009).

7 C. R. Jacob and M. Reiher, Int. J. Quantum Chem. 112, 3661 (2012).

${ }^{8}$ H. S. Yu, S. L. Li, and D. G. Truhlar, J. Chem. Phys. 145, 130901 (2016).

9 P. G. Szalay, T. Muller, G. Gidofalvi, H. Lischka, and R. Shepard, Chem. Rev. 112, 108 (2012).

10 J. W. Park, R. Al-Saadon, M. K. MacLeod, T. Shiozaki, and B. Vlaisavljevich, Chem. Rev. 120, 5878 (2020).

11 C. J. Cramer, M. Włoch, P. Piecuch, C. Puzzarini, and L. Gagliardi, J. Phys. Chem. A 110, 1991 (2006).

12 M. S. Gordon, D. G. Fedorov, S. R. Pruitt, and L. V. Slipchenko, Chem. Rev. 112, 632 (2012).

13 M. A. Collins and R. P. Bettens, Chem. Rev. 115, 5607 (2015).

${ }^{14}$ K. Raghavachari and A. Saha, Chem. Rev. 115, 5643 (2015).

15 D. G. Fedorov, Y. Alexeev, and K. Kitaura, J. Phys. Chem. Lett. 2, 282 (2011).

16 B. O. Roos, P. R. Taylor, and P. E. M. Siegbahn, Chem. Phys. 48, 157 (1980).

17 G. Li Manni, W. Dobrautz, N. A. Bogdanov, K. Guther, and A. Alavi, J. Phys. Chem. A 125, 4727 (2021).

18 P. P. Hallmen, H.-J. Werner, D. Kats, S. Lenz, G. Rauhut, H. Stoll, and J. van Slageren, Phys. Chem. Chem. Phys. 21, 9769 (2019).

19 P. Sharma, D. R. Pahls, B. L. Ramirez, C. C. Lu, and L. Gagliardi, Inorg. Chem. 58, 10139 (2019).

${ }^{20}$ R. W. Hogue, S. Singh, and S. Brooker, Chem. Soc. Rev. 47, 7303 (2018).
21 J. P. Malrieu, R. Caballol, C. J. Calzado, C. de Graaf, and N. Guihery, Chem. Rev. 114, 429 (2014).

${ }^{22}$ D. S. Levine, D. Hait, N. M. Tubman, S. Lehtola, K. B. Whaley, and M. Head-Gordon, J. Chem. Theory Comput. 16, 2340 (2020).

23 P. Sharma, V. Bernales, S. Knecht, D. G. Truhlar, and L. Gagliardi, Chem. Sci. 10, 1716 (2019).

${ }^{24}$ E. G. Hohenstein, N. Luehr, I. S. Ufimtsev, and T. J. Martínez, J. Chem. Phys. 142, 224103 (2015).

25 J. W. Snyder, B. F. Curchod, and T. J. Martínez, J. Phys. Chem. Lett. 7, 2444 (2016).

26 J. Olsen, B. O. Roos, P. Jørgensen, and H. J. A. Jensen, J. Chem. Phys. 89, 2185 (1988).

27 P.-Á. Malmqvist, A. Rendell, and B. O. Roos, J. Phys. Chem. 94, 5477 (1990).

28 D. Ma, G. L. Manni, and L. Gagliardi, J. Chem. Phys. 135, 044128 (2011).

29 J. Ivanic, J. Chem. Phys. 119, 9364 (2003).

30 S. M. Parker, T. Seideman, M. A. Ratner, and T. Shiozaki, J. Phys. Chem. C 118, 12700 (2014).

31 S. Nishio and Y. Kurashige, J. Chem. Phys. 151, 084111 (2019).

${ }^{32}$ R. K. Kathir, C. de Graaf, R. Broer, and R. W. A Havenith, J. Chem. Theory Comput. (2020), 10.1021/acs.jctc.9b01144.

${ }_{33}$ M. R. Hermes and L. Gagliardi, J. Chem. Theory Comput. 15, 972 (2019).

${ }^{34}$ R. Pandharkar, M. R. Hermes, C. J. Cramer, and L. Gagliardi, J. Phys. Chem. Lett. 10, 5507 (2019).

35 M. R. Hermes, R. Pandharkar, and L. Gagliardi, J. Chem. Theory Comput. 16, 4923 (2020).

36 R. Pandharkar, M. R. Hermes, C. J. Cramer, D. G. Truhlar, and L. Gagliardi, J. Chem. Theory Comput. 17, 2843 (2021).

37 D. I. Lyakh, M. Musiał, V. F. Lotrich, and R. J. Bartlett, Chem. Rev. 112, 182 (2012).

38 C. Møller and M. S. Plesset, Phys. Rev. 46, 618 (1934).

39 I. Shavitt and R. J. Bartlett, Many-Body Methods in Chemistry and Physics (Cambridge University Press, Cambridge, U.K., 2009).

${ }^{40}$ Y. Cao, J. Romero, J. P. Olson, M. Degroote, P. D. Johnson, M. Kieferová, I. D. Kivlichan, T. Menke, B. Peropadre, N. P. Sawaya, et al., Chem. Rev. 119, 10856 (2019). 
41 K. Head-Marsden, J. Flick, C. J. Ciccarino, and P. Narang, Chem. Rev. 121, 3061 (2020).

42 S. McArdle, S. Endo, A. Aspuru-Guzik, S. C. Benjamin, and X. Yuan, Rev. Mod. Phys. 92, 015003 (2020).

43 S. Lloyd, Science, 1073 (1996).

44 J. Romero, R. Babbush, J. R. McClean, C. Hempel, P. J. Love, and A. Aspuru-Guzik, Quantum Sci. Technol. 4, 014008 (2018).

45 A. Peruzzo, J. McClean, P. Shadbolt, M.-H. Yung, X.-Q. Zhou, P. J. Love, A. Aspuru-Guzik, and J. L. O'brien, Nat. Commun. 5, 1 (2014).

46 A. Y. Kitaev, arXiv preprint quant-ph/9511026 (1995).

47 D. S. Abrams and S. Lloyd, Phys. Rev. Lett. 83, 5162 (1999).

48 J. Preskill, Quantum 2, 79 (2018).

49 D. Wecker, M. B. Hastings, and M. Troyer, Phys. Rev. A 92, 042303 (2015).

50 J. R. McClean, M. E. Kimchi-Schwartz, J. Carter, and W. A. De Jong, Phys. Rev. A 95, 042308 (2017).

51 A. Kandala, A. Mezzacapo, K. Temme, M. Takita, M. Brink, J. M. Chow, and J. M. Gambetta, Nature 549, 242 (2017).

52 J. Olsen, B. O. Roos, P. Jo/rgensen, and H. J. A. Jensen, J. Chem. Phys. 89, 2185 (1988).

53 P. Å. Malmqvist, A. Rendell, and B. O. Roos, J. Phys. Chem. 94, 5477 (1990)

54 T. Fleig, J. Olsen, and C. M. Marian, J. Chem. Phys. 114, 4775 (2001).

55 D. Ma, G. Li Manni, and L. Gagliardi, J. Chem. Phys. 135, 044128 (2011).

56 J. Li, Y. Yao, A. A. Holmes, M. Otten, Q. Sun, S. Sharma, and C. Umrigar, Phys. Rev. Res. 2, 012015 (2020).

57 J. Li, M. Otten, A. A. Holmes, S. Sharma, and C. J. Umrigar, J. Chem. Phys. 149, 214110 (2018).

58 R. Olivares-Amaya, W. Hu, N. Nakatani, S. Sharma, J. Yang, and G. K.-L. Chan, J. Chem. Phys. 142, 034102 (2015).

59 S. Knecht, E. D. Hedegård, S. Keller, A. Kovyrshin, Y. Ma, A. Muolo, C. J. Stein, and M. Reiher, arXiv preprint arXiv:1512.09267 (2015), 10.2533/chimia.2016.244.

${ }^{60}$ Y. Kurashige and T. Yanai, J. Chem. Phys. 135, 094104 (2011).

${ }^{61}$ K. H. Marti and M. Reiher, in Progress in Physical Chemistry Volume 3 (Oldenbourg Wissenschaftsverlag, 2011) pp. 293-309.

62 A. Bernhardsson, R. Lindh, J. Olsen, and M. Fulscher, Mol. Phys. 96, 617 (1999).

63 J. Stålring, A. Bernhardsson, R. Lindh, J. Stalring, A. Bernhardssont, and R. Lindhf, Mol. Phys. 99, 103 (2001).

${ }^{64}$ Q. Sun and G. K. L. Chan, J. Chem. Theory Comput. 10, 3784 (2014).

65 S. McArdle, S. Endo, A. Aspuru-Guzik, S. C. Benjamin, and X. Yuan, Rev. Mod. Phys. 92, 015003 (2020).

${ }^{66}$ B. Bauer, S. Bravyi, M. Motta, and G. K.-L. Chan, Chem. Rev. 120, 12685 (2020).

${ }^{67}$ M. Murugesu, M. Habrych, W. Wernsdorfer, K. A. Abboud, and G. Christou, J. Am. Chem. Soc. 126, 4766 (2004), pMID: 15080666, https://doi.org/10.1021/ja0316824.

68 A. Baniodeh, N. Magnani, Y. Lan, G. Buth, C. E. Anson, J. Richter, M. Affronte, J. Schnack, and A. K. Powell, npj Quantum Mater. 3, 1 (2018).

${ }^{69}$ M. B. Smith and J. Michl, Annu. Rev. Phys. Chem. 64, 361 (2013).

70 D. Casanova, Chem. Rev. 118, 7164 (2018).

71 F. A. Evangelista, G. K.-L. Chan, and G. E. Scuseria, J. Chem. Phys. 151, 244112 (2019), https://doi.org/10.1063/1.5133059.

72 F. Arute, K. Arya, R. Babbush, D. Bacon, J. C. Bardin, R. Barends, S. Boixo, M. Broughton, B. B. Buckley, D. A. Buell, B. Burkett, N. Bushnell, Y. Chen, Z. Chen, B. Chiaro, R. Collins, W. Courtney, S. Demura, A. Dunsworth, E. Farhi,
A. Fowler, B. Foxen, C. Gidney, M. Giustina, R. Graff, S. Habegger, M. P. Harrigan, A. Ho, S. Hong, T. Huang, W. J. Huggins, L. Ioffe, S. V. Isakov, E. Jeffrey, Z. Jiang, C. Jones, D. Kafri, K. Kechedzhi, J. Kelly, S. Kim, P. V. Klimov, A. Korotkov, F. Kostritsa, D. Landhuis, P. Laptev, M. Lindmark, E. Lucero, O. Martin, J. M. Martinis, J. R. McClean, M. McEwen, A. Megrant, X. Mi, M. Mohseni, W. Mruczkiewicz, J. Mutus, O. Naaman, M. Neeley, C. Neill, H. Neven, M. Y. Niu, T. E. O’Brien, E. Ostby, A. Petukhov, H. Putterman, C. Quintana, P. Roushan, N. C. Rubin, D. Sank, K. J. Satzinger, V. Smelyanskiy, D. Strain, K. J. Sung, M. Szalay, T. Y. Takeshita, A. Vainsencher, T. White, N. Wiebe, Z. J. Yao, P. Yeh, and A. Zalcman, Science 369, 1084 (2020), https://www.science.org/doi/pdf/10.1126/science.abb9811.

73 A. J. McCaskey, Z. P. Parks, J. Jakowski, S. V. Moore, T. D. Morris, T. S. Humble, and R. C. Pooser, npj Quantum Inf. 5, 1 (2019).

${ }^{74}$ P. J. J. O’Malley, R. Babbush, I. D. Kivlichan, J. Romero, J. R. McClean, R. Barends, J. Kelly, P. Roushan, A. Tranter, N. Ding, B. Campbell, Y. Chen, Z. Chen, B. Chiaro, A. Dunsworth, A. G. Fowler, E. Jeffrey, E. Lucero, A. Megrant, J. Y. Mutus, M. Neeley, C. Neill, C. Quintana, D. Sank, A. Vainsencher, J. Wenner, T. C. White, P. V. Coveney, P. J. Love, H. Neven, A. AspuruGuzik, and J. M. Martinis, Phys. Rev. X 6, 031007 (2016).

75 H. R. Grimsley, S. E. Economou, E. Barnes, and N. J. Mayhall, Nat. Commun. 10, 1 (2019).

76 M. Cerezo, A. Arrasmith, R. Babbush, S. C. Benjamin, S. Endo, K. Fujii, J. R. McClean, K. Mitarai, X. Yuan, L. Cincio, et al., Nat. Rev. Phys. , 1 (2021).

77 K. Bharti, A. Cervera-Lierta, T. H. Kyaw, T. Haug, S. AlperinLea, A. Anand, M. Degroote, H. Heimonen, J. S. Kottmann, T. Menke, et al., arXiv preprint arXiv:2101.08448 (2021).

78 D. A. Fedorov, Y. Alexeev, S. K. Gray, and M. Otten, arXiv preprint arXiv:2109.12652 (2021).

79 P. W. Shor, SIAM Review 41, 303 (1999).

80 A. W. Harrow, A. Hassidim, and S. Lloyd, Phys. Rev. Lett. 103, 150502 (2009).

81 P. Shor, in Proceedings 35th Annual Symposium on Foundations of Computer Science (1994) pp. 124-134.

82 D. Coppersmith, (2002).

83 G. Ortiz, J. E. Gubernatis, E. Knill, and R. Laflamme, Phys. Rev. A 64, 022319 (2001).

${ }^{84}$ R. Babbush, J. McClean, D. Wecker, A. Aspuru-Guzik, and N. Wiebe, Phys. Rev. A 91, 022311 (2015).

85 D. W. Berry, A. M. Childs, R. Cleve, R. Kothari, and R. D. Somma, Phys. Rev. Lett. 114, 090502 (2015).

${ }^{86}$ G. H. Low and I. L. Chuang, Quantum 3, 163 (2019).

87 P. Jordan and E. P. Wigner, in The Collected Works of Eugene Paul Wigner (Springer, 1993) pp. 109-129.

${ }^{88}$ S. Bravyi, J. M. Gambetta, A. Mezzacapo, and K. Temme, arXiv preprint arXiv:1701.08213 (2017).

89 S. B. Bravyi and A. Y. Kitaev, Ann. Phys. 298, 210 (2002).

90 V. E. Elfving, B. W. Broer, M. Webber, J. Gavartin, M. D. Halls, K. P. Lorton, and A. Bochevarov, arXiv preprint arXiv:2009.12472 (2020).

91 H. Liu, G. H. Low, D. S. Steiger, T. Häner, M. Reiher, and M. Troyer, arXiv preprint arXiv:2102.10081 (2021).

92 I. H. Kim, E. Lee, Y.-H. Liu, S. Pallister, W. Pol, and S. Roberts, arXiv preprint arXiv:2104.10653 (2021).

93

94 A. G. Taube and R. J. Bartlett, Int. J. Quantum Chem. 106, 3393 (2006).

95 W. Kutzelnigg, Theor. Chim. Acta 80, 349 (1991). 
96 Y. Shen, X. Zhang, S. Zhang, J.-N. Zhang, M.-H. Yung, and K. Kim, Phys. Rev. A 95, 020501 (2017).

97 G. Harsha, T. Shiozaki, and G. E. Scuseria, J. Chem. Phys. 148, 044107 (2018), https://doi.org/10.1063/1.5011033.

98 M. R. Hermes, "https://github.com/MatthewRHermes/mrh," (2018).

99 J. Chen, H.-P. Cheng, and J. Freericks, J. Chem. Theory Comput. 17, 841 (2021), arXiv:2008.06637.
100 K. Svore, A. Geller, M. Troyer, J. Azariah, C. Granade, B. Heim, V. Kliuchnikov, M. Mykhailova, A. Paz, and M. Roetteler, in Proceedings of the Real World Domain Specific Languages Workshop 2018 (2018) pp. 1-10.

101 G. H. Low, N. P. Bauman, C. E. Granade, B. Peng, N. Wiebe, E. J. Bylaska, D. Wecker, S. Krishnamoorthy, M. Roetteler, K. Kowalski, et al., arXiv preprint arXiv:1904.01131 (2019). 